\title{
APPRENTISSAGE ACTIF FONDÉ SUR LA CLASSE INVERSÉE DANS UN COURS DE GÉNIE MÉCANIQUE
}

\author{
Patrick Terriault \\ École de technologie supérieure \\ patrick.terriault@etsmtl.ca
}

\begin{abstract}
Résumé - Cet article présente un projet de recherche dont le but est d'étudier l'efficacité de la classe inversée implantée dans un cours obligatoire de génie mécanique. L'inversion du cours a été motivée par la passivité des étudiants durant les séances de cours magistraux et par les apprentissages en surface qu'ils réalisent. Pour remédier à cette problématique, le cours a été inversé en prenant en considération les déterminants de la motivation, certains éléments de la psychologie cognitive ainsi que la taxonomie de Bloom. Après avoir développé du nouveau matériel pédagogique, la classe inversée a été testée pour la première fois à l'hiver 2019. Les résultats préliminaires sont encourageants, car les étudiants se sont montrés satisfaits du cours lorsqu'ils se sont prononcés par un court questionnaire, par l'évaluation institutionnelle et lors d'un entretien de groupe. Les travaux se poursuivent vers une étude rigoureuse qui, à l'automne 2020, comparera l'efficacité d'un même cours offert en classe traditionnelle et en classe inversée.
\end{abstract}

Mots-clés : Apprentissage actif, classe inversée, motivation, psychologie cognitive, taxonomie de Bloom.

\section{INTRODUCTION}

Depuis son embauche comme professeur régulier à l'École de technologie supérieure (ÉTS) en 1999, le professeur Terriault a adopté une posture transmissive dans sa tâche d'enseignement en ayant recours à des exposés magistraux. Depuis quelques années, comme le soulignent Dumont et Berthiaume dès le second paragraphe de leur ouvrage [5], cette méthode d'enseignement classique ne semble plus être adaptée aux étudiants d'aujourd'hui. De plus, toujours selon ces mêmes auteurs, avec toute l'information disponible sur Internet et accessible instantanément à tout moment et en tout lieu, le professeur n'est donc plus le seul à détenir le savoir. Ces constats conduisent à une problématique qui se manifeste de deux manières :

i) Les étudiants sont de plus en plus passifs durant les séances de cours de telle sorte que les interactions entre eux et le professeur sont réduites au minimum. Les questions spontanées qui permettent au professeur de décoder les problèmes de compréhension et d'entamer un dialogue constructif avec les étudiants sont devenues rarissimes. Pour le professeur, la tâche d'aller donner un cours où l'interaction avec les étudiants est réduite au minimum est plutôt démotivante. L'expertise du professeur n'est pas réellement exploitée et ce dernier a l'impression de ne plus faire adéquatement son travail [9].

ii) Les étudiants ne semblent pas être en mesure de transposer adéquatement les compétences développées dans un cours vers un autre cours, notamment dans leur projet de fin d'études. Une proportion significative d'étudiants semble donc avoir opté pour un apprentissage en surface dont le but est de simplement obtenir la note de passage au cours, plutôt que de viser un apprentissage en profondeur afin de développer des compétences utiles pour l'avenir [7].

Devant cette problématique, la conception de l'enseignement et de l'apprentissage du professeur Terriault a été modifiée pour améliorer la formation des étudiants et pour retrouver la passion d'enseigner. Cependant, les choses doivent être faites différemment en confrontant les étudiants à un mode d'apprentissage actif au lieu de les exposer à un enseignement magistral transmissif. La classe inversée [5, 14] semble donc représenter une avenue prometteuse à explorer.

Cet article aborde l'utilisation de la classe inversée dans un cours de génie mécanique offert à l'ÉTS. L'étude de l'efficacité de la classe inversée fait l'objet d'un projet de recherche qui sera déployé jusqu'en 2021 et qui sera réalisé en différentes étapes. Considérant que l'inversion d'un cours enseigné en classe traditionnelle vers la classe inversée nécessite un investissement important de temps et d'effort de la part du professeur, le but du projet de recherche est de mesurer la véritable efficacité de la classe inversée de manière la plus objective possible. Des travaux similaires ont été réalisés dans le passé [11], mais certaines conditions rendent difficile la transposition des résultats (par exemple en comparant une classe traditionnelle et une classe inversée offertes à des trimestres différents ou qui font intervenir du personnel enseignant différent). Le but du présent de recherche est donc d'étudier l'efficacité de la classe inversée en comparant un groupe expérimental (classe inversée) et un 
groupe témoin (classe traditionnelle) soumis au plus grand nombre d'invariants possibles, notamment, le trimestre, le professeur, le chargé de travaux pratiques, les examens, le matériel pédagogique, etc.

Le but de cet article est de présenter les grandes lignes du projet de recherche et de faire état des résultats préliminaires obtenus jusqu'à présent suite à l'inversion d'un cours obligatoire de $1^{\text {er }}$ cycle du programme de génie mécanique de l'ÉTS.

Après avoir présenté le contexte, notamment le portait de l'ÉTS, du programme de génie mécanique et du cours faisant l'objet de cette publication, le cadre théorique sur lequel repose la classe inversée sera discuté. Suivront ensuite une brève présentation de la classe inversée et les grandes étapes du projet de recherche. Enfin, avant la conclusion, les résultats préliminaires obtenus à l'hiver 2019 suite à la première expérience de la classe inversée seront exposés.

\section{CONTEXTE}

Cette étude est réalisée au sein du Département de génie mécanique de l'École de technologie supérieure (ÉTS), un des dix établissements qui compose le réseau de l'Université du Québec. Situé à Montréal, l'ÉTS a vu le jour en 1974 pour former de technologues et en 1989, sa vocation a été modifiée pour devenir une école d'ingénieurs. Malgré son jeune âge, l'ÉTS est aujourd'hui la deuxième plus grande école ou faculté d'ingénierie au Canada en ce qui a trait au nombre d'étudiants au $1^{\text {er }}$ cycle. L'ÉTS est une université monofacultaire qui forme exclusivement des ingénieurs. La majorité des étudiants provient de cégeps québécois. En effet, $62 \%$ des étudiants de $1^{\text {er }}$ cycle sont issus d'un programme collégial technique (trois années de cégep après les études secondaires) et $27 \%$ d'un programme collégial en sciences de la nature (deux années de cégep après les études secondaires). Ces derniers doivent cependant suivre une année de formation technique à l'ÉTS avant d'entamer leurs études dans un des sept baccalauréats en ingénierie.

La clientèle de l'ÉTS est particulière, car elle est majoritairement composée de techniciens qui peuvent à tout moment quitter l'université pour occuper un emploi sur le marché du travail. Ces étudiants sont donc pragmatiques et généralement motivés à terminer leurs études universitaires rapidement. Puisque la première mission de l'Université du Québec qui est d'accroître le niveau de formation de la population québécoise par une accessibilité au savoir et à la culture, aucun contingentement n'est appliqué à l'ÉTS. Ainsi, les diplômés d'un programme collégial admissible peuvent débuter leurs études à l'ÉTS, et cela indépendamment de leurs résultats académiques antérieurs.

Le programme de baccalauréat en génie mécanique de l'ÉTS compte 115 crédits, auxquels s'ajoute la réussite de trois stages rémunérés en industrie d'une durée d'au moins quatre mois chacun. Les étudiants peuvent partir en stage à l'hiver (de janvier à avril), à l'été (de mai à août) ou à l'automne (de septembre à décembre). Environ 1050 étudiants sont présentement actifs dans le programme et 94\% d'entre eux sont des hommes. Les cours sont offerts à des groupes dont la taille est relativement petite (entre 30 et 60 étudiants). Par conséquent, les cours obligatoires du programme s'offrent entre huit à dix fois par année, et cela à chacune des trois sessions afin d'accommoder les étudiants qui partent en stage à tout moment de l'année.

Le cours dans lequel la classe inversée est déployée s'intitule «MEC423 - Méthode éléments finis des corps déformables ». Il est généralement suivi à la $4^{\mathrm{e}}$ session, parfois à la $5^{\mathrm{e}}$, d'un cheminement qui en compte huit (excluant les sessions de stage). Il s'agit d'un cours obligatoire de 4 crédits pour lequel la charge de travail attendue est de 12 heures par semaine (trois heures de cours, trois heures de travaux pratiques et six heures de travail libre à la maison). La principale compétence développée dans ce cours concerne l'analyse et la conception de pièces supportant des charges mécaniques et thermiques avec la méthode des éléments finis.

\section{CADRE THÉORIQUE}

Pour maximiser les chances de réussir l'inversion d'un cours, un certain nombre de concepts théoriques sont pris en considération, notamment la motivation étudiante, la psychologie cognitive et la taxonomie de Bloom.

En ce qui a trait à la motivation étudiante, la théorie de l'autodétermination proposée par Abeysekera et Dawson [1] concerne essentiellement la motivation qui peut se manifester de manière intrinsèque ou extrinsèque. La motivation intrinsèque est le désir d'un individu à apprendre, à explorer et à élargir ses horizons lorsqu'il est confronté à de nouveaux apprentissages [12]. La motivation intrinsèque s'apparente donc au concept d'apprentissage en profondeur dont le but fondamental est d'élargir ses compétences. La motivation extrinsèque est le désir d'individu à réaliser une tâche pour obtenir une récompense ou pour éviter une pénalité. Un tel comportement mène généralement à des apprentissages en surface qui sont rapidement oubliés une fois l'évaluation terminée. En considérant également les travaux de Viau [16], quatre déterminants de la motivation intrinsèque se dégagent : i) l'étudiant possède les compétences requises pour mener à terme une activité pédagogique, ii) l'étudiant exerce un contrôle sur le déroulement de l'activité, iii) l'étudiant perçoit qu'une activité à une

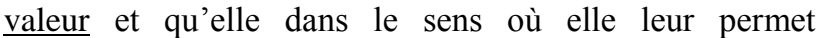
d'atteindre ses objectifs personnels, et iv) l'étudiant a un besoin d'appartenir à un groupe afin de pouvoir interagir avec ses pairs, ce qui est appelé l'interdépendance.

Selon les fondements de la psychologie cognitive [6], l'apprentissage survient lorsque de nouvelles informations 
passent de la mémoire de travail à la mémoire à long terme après avoir été encodées. Cependant, pour que le processus d'apprentissage soit efficace, il est donc essentiel de ne pas exposer les étudiants à trop de nouvelles informations simultanément. Si cela devait s'avérer, certaines informations seraient simplement évacuées sans avoir été assimilées. C'est ce qui est appelé la surcharge cognitive. Il est donc important que le professeur soit conscient de cette réalité et qu'il conçoive des activités pédagogiques qui exposent les nouveaux contenus aux étudiants à un rythme optimal. Un autre concept important de la psychologie cognitive est la zone proximale de développement [17]. Ce concept, selon lequel une nouvelle information peut être assimilée seulement si elle est suffisamment "près" des connaissances antérieurement acquises, impose donc au professeur de bien planifier, découper et séquencer les apprentissages des étudiants pour qu'ils se situent constamment dans leur zone proximale de développement. Enfin, la théorie de la charge cognitive proposée par Sweller [13] concerne la gestion de la mémoire de travail en définissant trois types de charges cognitives : intrinsèques, extrinsèques et accessoires. Une charge cognitive intrinsèque est l'information (concept, notion, théorie, etc.) proprement dite que l'étudiant doit assimiler. Cette charge cognitive ne peut pas être réduite et elle doit donc se situer dans la zone proximale de développement de l'étudiant, sans quoi il lui sera impossible de l'encoder adéquatement. Une charge cognitive extrinsèque est une notion non essentielle (par exemple une longue mise en contexte plus ou moins pertinente pour introduire un concept) qui s'ajoute inutilement dans le processus d'apprentissage et qui représente une nuisance. Ces charges extrinsèques peuvent être retirées pour favoriser l'efficacité de l'apprentissage. Enfin, une charge cognitive accessoire est un ajout (comme un exemple, un exercice, une démonstration, etc.) qui aide l'étudiant à assimiler une charge cognitive intrinsèque.

Enfin, la taxonomie de Bloom propose une classification des processus mentaux qui interviennent dans les activités d'apprentissage auxquelles sont confrontés les étudiants. La taxonomie, d'abord proposée par Bloom dans les années 1950, a été révisée par Krathwohl [8]. Dans la taxonomie révisée, les processus mentaux liés à l'apprentissage sont classés en six niveaux, allant du niveau le plus élémentaire jusqu'au niveau le plus exigeant : 1-Connaissances, 2-Compréhension, 3-Application, 4-Analyse, 5-Évaluation et 6-Création.

\section{CLASSE INVERSÉE}

Le qualificatif « inversée » est employé en opposition au modèle traditionnel qu'est l'enseignement magistral transmissif. Dans une classe traditionnelle, les étudiants assistent à des exposés magistraux offerts par le professeur tout en prenant des notes. Sauf lorsqu'il y a des questions suite auxquelles le professeur entame une discussion, les étudiants sont plutôt passifs et les activités qui s'y déroulent sont généralement de faible niveau cognitif (1-Connaitre, 2-Comprendre ou 3-Appliquer). Les activités de plus haut niveau cognitif (4-Analyser, 5-Critiquer ou 6-Créer) sont ensuite réalisées à la maison par l'étudiant, sans que ce dernier ait accès au professeur en cas de difficulté. Simplement dit, le modèle traditionnel consiste donc à faire d'abord la théorie en classe et ensuite la pratique à la maison. La figure 1 schématise le déroulement d'une semaine d'activité typique du cours MEC423 offert en classe traditionnelle. Les deux principaux problèmes avec cette formule sont : i) la matière est présentée au même rythme pour tous les étudiants, ce qui risque de surcharger cognitivement certains d'entre eux, et ii) les processus cognitifs que les étudiants doivent déployer en présence du professeur sont de bas niveau cognitif (comprendre et appliquer), tandis que les processus de haut niveau cognitif sont déployés à la maison en absence du professeur. En parallèle, la séance hebdomadaire de travaux pratiques est essentiellement consacrée à l'apprentissage du logiciel d'analyse par éléments finis ANSYS.

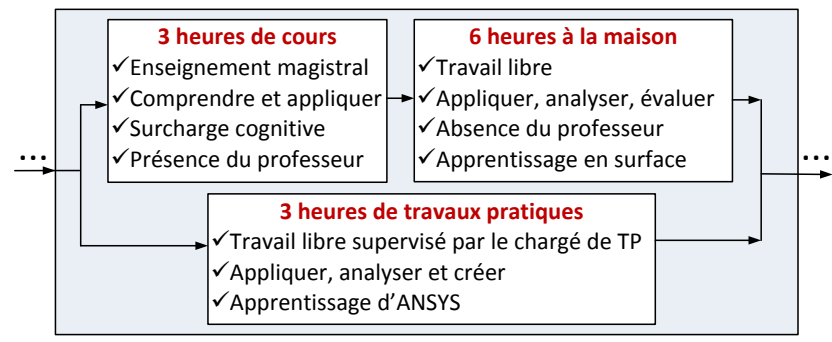

Fig. 1. Semaine typique de classe traditionnelle.

La classe inversée vise justement à inverser cette réalité en impliquant d'abord l'étudiant dans des activités de bas niveau taxonomique avant un cours alors qu'il est seul à la maison, et ensuite en lui faisant vivre des activités de haut niveau taxonomique dans la classe en présence du professeur. L'expertise du professeur peut alors être judicieusement exploitée. On pourrait ainsi vulgariser la classe inversée en stipulant que la théorie est d'abord vue à la maison et la pratique s'effectue ensuite en classe.

Dans la phase d'autoapprentissage à la maison, chaque étudiant apprend à son propre rythme tout en ayant la possibilité de prendre des pauses pour un repos cognitif ou de revenir en arrière sur certaines notions mal comprises. Ces activités se déroulent à la maison dans un environnement plus chaleureux et moins contraignant qu'un lieu public. Dans la phase de pédagogie active en classe, davantage de temps est dégagé pour le professeur afin d'aborder des activités qui requièrent un engagement cognitif élevé. De plus, en favorisant le travail en équipe, les étudiants ayant le plus de facilité peuvent aider ceux qui apprennent moins rapidement ou qui éprouvent des 
difficultés, ce qui favorise l'interdépendance. Enfin, l'étudiant ne va pas acquérir plus de connaissances dans la classe inversée que dans une classe traditionnelle, mais il y aura un apprentissage plus en profondeur, car c'est lui qui aura construit ses connaissances.

Malheureusement, peu d'études ont été réalisées pour mesurer l'influence de la classe inversée sur les résultats académiques des étudiants. Comme le souligne Tricot [15], le nombre d'études contrôlées où l'on compare les résultats d'étudiants impliqués dans un dispositif de classe inversée avec ceux ouvrant dans un dispositif d'enseignement traditionnel est marginal.

\section{PRÉSENTATION DU PROJET}

Un projet de recherche a donc été entrepris pour étudier de manière la plus objective possible la véritable efficacité de la classe inversée. Le projet est déployé en trois étapes successives.

\section{1. Étape I - Développement du matériel}

Auparavant, le cours était offert en classe traditionnelle magistrale et les étudiants devaient acheter un livre de référence en anglais. Puisque le contenu du livre n'était pas entièrement adapté aux objectifs pédagogiques du cours, le professeur devait apporter des nuances durant les exposés magistraux et compléter le contenu du cours fournissant aux des étudiants des compléments (copie de diapositives, notes de cours, etc.). En plus du prix relativement élevé du manuel de référence, l'ouvrage est rédigé en anglais, ce qui représente une charge cognitive extrinsèque importante pour de nombreux étudiants.

Pour favoriser l'inversion du cours et pour favoriser l'autonomie des étudiants lors de la lecture du contenu théorique, des cahiers ont été rédigés durant les années 2017 et 2018. Ces cahiers pourraient éventuellement être regroupés pour former les chapitres d'un livre. Les cahiers ont été rédigés en français de manière à ce que le contenu ne représente que des charges cognitives intrinsèques (nouvelles notions proprement dites) et accessoires (présentation d'exemples d'application détaillés). Des exercices couvrant l'entièreté du contenu ont été préparés et présentés à la fin des cahiers.

En plus des cahiers, un billet d'entrée a été préparé pour chaque séance de cours. Le billet d'entrée est en quelque sorte un devoir à faire avant un cours formulé pour que l'étudiant demeure dans sa zone proximale de développement. L'étudiant doit effectuer une tâche, généralement la résolution d'un exercice simple, qui se veut une première application de la nouvelle matière. Les billets d'entrée comptent pour $20 \%$ de la note finale. Durant la session, les étudiants devaient remettre 11 billets d'entrée et chacun d'eux valait 2 points, ce qui totalise 22 points. Cependant, seulement les premiers 20 points sont comptabilisés, ce qui revient à dire qu'un étudiant peut, sans pénalité, choisir de ne pas remettre un billet d'entrée, ou encore que les premières erreurs sont sans conséquence.

\section{2. Étape II - Rodage de la classe inversée}

Avant d'étudier l'efficacité de la classe inversée, il a été jugé nécessaire de roder le cours MEC423 en classe inversée à au moins deux reprises avant de comparer cette formule pédagogique à un enseignent traditionnel qui est peaufiné depuis 2007. Par conséquent, le cours MEC423 a été offert en classe inversée à trois groupes à l'hiver 2019 et il le sera à deux autres groupes à l'automne 2019. Les principaux constats de l'expérience vécue à l'hiver 2019 seront abordés dans la prochaine section de cet article. La figure 2 schématise le déroulement d'une semaine typique de cours en classe inversée. Avant une séance de cours, l'étudiant doit lire un ou deux cahiers à la maison afin d'établir un premier contact avec le nouveau contenu. Puisque l'étudiant avance à son propre rythme, les risques qu'il se retrouve en surcharge cognitive sont minimisés. Il n'est pas attendu que l'étudiant soit en mesure de tout assimiler adéquatement suite à la lecture des cahiers, mais bien qu'il soit en mesure de résoudre le billet d'entrée (un exercice simple en lien avec la nouvelle matière). L'étudiant ne doit pas résoudre les exercices à la fin des cahiers.

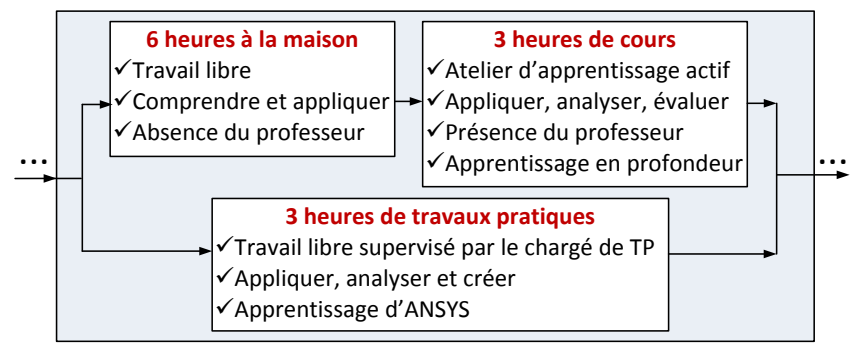

Fig. 2. Semaine typique de classe inversée.

En se présentant au cours, l'étudiant remet son billet d'entrée en le déposant sur le bureau du professeur. Le cours début ensuite par un bref survol de la nouvelle matière présentée dans les cahiers. Ce survol, effectué de manière magistrale, ne remplace d'aucune façon la lecture préalable des cahiers. Il permet au professeur d'insister sur les points essentiels tout en offrant la possibilité aux étudiants de demander des clarifications sur les aspects les moins bien assimilés. Dépendant du nombre de questions, le survol peut nécessiter entre 15 et 45 minutes. Par la suite, le professeur distribue les billets d'entrée aux étudiants de la classe et commente la solution pendant que les étudiants procèdent à la correction du billet d'entrée d'un camarade de classe. Pour le reste du cours dont la durée varie entre 135 et 165 minutes, les étudiants se regroupent en équipe pour résoudre les exercices à la fin des cahiers. Ce processus d'apprentissage actif, appelé atelier de résolution d'exercices, permet aux étudiants d'assimiler les concepts plus avancés abordés dans les 
cahiers. En cas de difficulté, le professeur intervient auprès d'une seule équipe, voire même de la classe entière, pour apporter au besoin des clarifications. C'est lors des ateliers de résolution d'exercices que les étudiants sont appelés à déployer des processus cognitifs de plus haut niveau taxonomique, notamment en analysant en profondeur des problèmes et en évaluant leur démarche de résolution. Le fait de pouvoir échanger avec leurs pairs et de profiter de la présence du professeur en cas de difficultés favorise un apprentissage en profondeur.

\section{3. Étape III - Étude comparative}

Après avoir rodé la formule de classe inversée lors des sessions d'hiver et d'automne 2019, l'étude comparative pour évaluer son efficacité sera réalisée à l'automne 2020. Le cours MEC423 sera alors sous la responsabilité du même professeur, en utilisant le même matériel pédagogique et en proposant les mêmes évaluations, mais à deux groupes différents : le premier groupe en classe traditionnelle et le second groupe en classe inversée. L'efficacité de la classe inversée sera évaluée en comparant plusieurs indicateurs :

- La performance académique des étudiants mesurée par les notes aux différentes évaluations (examens, projet, etc.),

- Le niveau d'engagement cognitif durant les séances de cours mesuré à partir de capteurs électrodermaux installés sur quelques étudiants,

- La motivation des étudiants mesurée à partir de questionnaires ou d'entretiens,

- La satisfaction du cours mesurée à partir de l'évaluation institutionnelle réalisée à la fin de la session.

Tous ces indicateurs seront analysés afin de tirer des conclusions sur l'efficacité de la classe inversée.

\section{RÉSULATS PRÉLIMINAIRES}

Au moment d'écrire cet article, l'étape I du projet de recherche est complétée. Les cahiers ont été rédigés et ils totalisent plus de 250 pages. Les billets d'entrée ont également été préparés et mis en application au courant de l'hiver 2019. L'étape II est en cours de réalisation. La session d'hiver 2019 est terminée et la classe inversée a été déployée dans trois groupes auprès de 117 étudiants.

À l'hiver 2019, les étudiants ont été sondés afin de connaitre leurs impressions ainsi que la façon dont ils vivent la classe inversée. À la $6^{\mathrm{e}}$ semaine de cours, ils ont été invités à prendre une feuille vierge et à écrire leurs impressions, énoncer leurs perceptions ou décrire leur expérience par rapport à la classe inversée dans laquelle ils ont œuvré depuis le début de la session. Il s'agit donc d'un court questionnaire composé d'une seule question à laquelle est attendue une réponse prenant la forme d'un texte d'environ une demi-page. Certains étudiants n'ont écrit qu'une seule phrase, tandis que d'autres ont dépassé la demi-page. Les étudiants devaient participer sur une base volontaire et ils ne devaient pas écrire leur nom afin que les réponses demeurent anonymes. Les étudiants ont disposé d'environ quinze minutes pour répondre. À cause d'une tempête de neige qui a forcé l'annulation d'un cours, seulement deux des trois groupes ont pu être sondés et 55 réponses ont été collectées. L'objectif était de collecter des données de manière inductive [3].

Les données ont été analysées et regroupées en commentaires positifs ou négatifs. Dans un souci de concision, seulement les commentaires qui ont été soulevés par plus d'un étudiant sont rapportés ici. Certains étudiants ont parlé du contenu du cours, de sa pertinence ou des installations physiques dans lesquelles les cours se déroulent, sans aborder la classe inversée proprement dite. Ces commentaires ont également été retirés. Le chiffre entre parenthèses indique le nombre de fois que le commentaire a été soulevé.

- Commentaires positifs :

- Les étudiants sont à jour dans la matière (17),

- La classe inversée permet aux étudiants de mieux comprendre la matière (15),

- Les cahiers sont clairs et bien rédigés, ce qui aide à la compréhension de la matière (8),

- Les ateliers de résolution d'exercices sont stimulants/motivants/intéressants (6),

- La classe inversée développe l'autoformation (3).

- Commentaires négatifs :

- Les billets d'entrée ne devraient pas être évalués ni compter dans la note finale (5),

- La remise et la correction en classe des billets d'entrée rendent obligatoire la présence au cours (4),

- Il faut investir plus de temps dans ce cours que dans les autres cours (4),

- La matière devrait être enseignée en classe, et non pas seulement présentée dans les cahiers (3),

- Les billets d'entrée devraient être remplacés par des devoirs à faire après les cours (2).

Un premier constat suite à l'analyse de ces données qualitatives indique qu'un nombre plus élevé de commentaires positifs ont été formulés. Ceci est encourageant, car les étudiants semblent adhérer majoritairement à la classe inversée.

En ce qui concerne les commentaires positifs, le plus fréquent d'entre eux est le fait que la classe inversée a forcé les étudiants à être à jour dans leurs apprentissages. Cependant, ce n'est nécessairement la classe inversée qui oblige les étudiants à être à jour, mais bien le fait qu'un billet d'entrée doit être rendu chaque semaine. Dans une classe traditionnelle, un devoir à remettre après chaque séance de cours aurait probablement le même effet. Le second commentaire le plus fréquent concerne la qualité de l'apprentissage et il est permis de croire que la classe inversée est en la cause. Le troisième commentaire positif souligne la qualité des cahiers qui auraient pu avoir été développés dans un contexte de classe traditionnelle. Cependant, les cahiers ont été conçus et rédigés dans le 
but de faciliter l'apprentissage des étudiants de manière autonome. L'initiative semble donc porter fruit. Le quatrième commentaire positif concerne le déroulement des séances de cours. Plusieurs étudiants semblent apprécier les ateliers de résolution d'exercices en équipe, ce qui a été rendu possible dans un contexte de classe inversée. Finalement, quelques étudiants ont apprécié le fait que l'autoformation est favorisée. Cet aspect inattendu est néanmoins fort intéressant, car il peut être relié à la $12^{\mathrm{e}}$ qualité requise des diplômes que les programmes d'ingénierie doivent développer selon le Bureau canadien d'agrément de programmes de génie.

Pour ce qui est des commentaires négatifs, il semble évident que certains étudiants préfèrent un mode d'enseignement magistral transmissif. En effet, des étudiants n'ont pas apprécié devoir se présenter aux séances de cours pour participer à la correction des billets d'entrée et pour recevoir les points qui leur sont associés ( $2^{\mathrm{e}}$ commentaire négatif). De plus, certains démontrent une vision très traditionnelle de l'enseignement selon laquelle un apprentissage doit se faire de manière magistrale en assistant à la prestation du professeur en classe $\left(4^{\mathrm{e}}\right.$ commentaire négatif) et en étant évalué après cette prestation $\left(5^{\mathrm{e}}\right.$ commentaire négatif). Les deux autres commentaires négatifs requièrent plus de réflexion. Le $1^{\text {er }}$ commentaire négatif soulève la question de l'évaluation des billets d'entrée. Ces derniers sont considérés par le professeur comme des évaluations formatives qui, idéalement, ne devraient pas être comptabilisées dans le calcul de la note finale. Par contre, s'ils étaient strictement formatifs, il y a fort à parier que les étudiants ne les feraient pas. En effet, même en ayant un caractère sommatif, les billets d'entrée n'ont pas été systématiquement remis par tous les étudiants. En fait, il y avait au total 11 billets d'entrée à remettre et en moyenne, les étudiants en ont remis 9,3. La note moyenne pour l'ensemble des billets d'entrée a été 15,8/20. Même si les exercices proposés dans les billets d'entrée étaient plutôt simples, certains étudiants n'ont pas été en mesure de bien les réaliser. Une explication possible ne concerne pas le niveau de difficulté des exercices traités dans les billets d'entrée, mais bien l'investissement de temps accru que requiert la classe inversée. En effet, comme le rapporte le $3^{\mathrm{e}}$ commentaire négatif, certains étudiants ont indiqué avoir investi plus de temps dans ce cours que dans les autres cours. Ceci n'est pas surprenant, car l'apprentissage s'effectue principalement de manière individuelle à la maison et au propre rythme de l'étudiant. Puisque que l'étudiant apprend par lui-même, le risque d'un apprentissage en profondeur est augmenté, mais au détriment d'un investissement de temps plus important.

Un entretien de groupe [2] a ensuite été organisé pour chercher à comprendre encore plus l'expérience vécue par les étudiants. Les étudiants des trois groupes ont été invités à participer à un entretien d'environ une heure pour échanger sur leur expérience avec la classe inversée.
Seulement trois d'entre eux se sont portés volontaires. Ils ont été regroupés dans une salle de réunion et des enregistrements audio et vidéo ont été réalisés dans le but d'écrire le verbatim de l'entretien. Une fois celui-ci rédigé, les enregistrements ont été détruits.

Le verbatim a ensuite été analysé dans une perspective inductive délibérative [10]. Le cadre théorique a donc servi à dégager les codes et le logiciel NVivo 12 a été utilisé pour procéder au codage. Ces codes et le nombre de références sont fournis au tableau 1 .

Tableau 1 : Codes employés dans l'analyse du verbatim.

\begin{tabular}{|l|r|}
\hline Code & Références \\
\hline A. Motivation & 30 \\
\hline A.1 Intrinsèque & \multicolumn{2}{|c|}{17} \\
\hline A.1.1 Compétence & \multicolumn{2}{r|}{} \\
\hline A.1.2 Contrôle & \multicolumn{2}{r|}{} \\
\hline A.1.3 Interdépendance & \multicolumn{2}{|c|}{4} \\
\hline A.1.4 Valeur & \multicolumn{2}{|c|}{13} \\
\hline A.2 Extrinsèque & 17 \\
\hline B. Psychologie cognitive & \multicolumn{2}{|c|}{2} \\
\hline B.1 Surcharge cognitive & \multicolumn{2}{|c|}{7} \\
\hline B.2 Zone proximale de développement & \multicolumn{2}{|c|}{8} \\
\hline B.3 Types de charges cognitives & 11 \\
\hline C. Taxonomie de Bloom & 24 \\
\hline D. Matériel résiduel & \multicolumn{2}{|l}{} \\
\hline
\end{tabular}

Les quatre déterminants de la motivation intrinsèque ont émergé lors de l'entretien de groupe et l'interdépendance est celui qui a été le plus souvent abordé. Les étudiants interviewés ont apprécié pouvoir interagir avec leurs pairs durant les ateliers de résolution d'exercices comme en témoigne un participant :
"Quand on travaille à résoudre des exercices, je consulte seulement les personnes qui sont à mes côtés. Le climat est agréable, car tu peux échanger avec tes voisins. Même si la disposition de la classe n'est pas propice à faire du travail en équipe, il est quand même facile de communiquer avec les gens autour de soi et de finalement faire du travail en équipe. »

Contrairement à ce qui était initialement espéré, le fait de corriger la copie du billet d'entrée d'un autre étudiant ne s'avère pas être une stratégie qui contribue à un apprentissage en profondeur :

\section{"Quand tu corriges le billet d'entrée d'un autre, tu constates que la personne a fait une erreur dans la solution, mais tu ne verras pas une méthode différente de la tienne. Je ne pense pas que ce soit formateur pour nous. »}

Il est donc pertinent de remettre en question la correction des billets d'entrée par les paris afin de dégager plus de temps pour l'atelier de résolution d'exercices en 
équipe. Pour ce qui est de la motivation extrinsèque, les étudiants interviewés ont déploré qu'un nombre significatif de leurs pairs ne prennent pas au sérieux leurs études et que leur seul objectif soit de simplement passer le cours. Ceci pourrait expliquer les réticences envers la classe inversée qui ont émergé lors de l'analyse du questionnaire. Un participant a même brossé un portrait plutôt sombre de la situation en estimant qu'environ $40 \%$ des étudiants étaient motivés extrinsèquement :

"Il y a un peu plus de gens qui font des études pour avoir des connaissances, mais il y en a trop qui ne veulent qu'avoir un diplôme. En fait, je dirais qu'il y a environ $60 \%$ de gens consciencieux, et $40 \%$ paresseux. »

Les données actuellement disponibles ne permettent pas d'étudier rigoureusement cette question qui pourrait pourtant avoir un impact important sur le succès de l'implantation de la classe inversée. Si une proportion trop élevée d'étudiants n'est motivée qu'extrinsèquement, une implantation réussie de la classe inversée risque donc d'être compromise. Cette question mérite d'être approfondie au courant des prochains mois.

L'analyse du verbatim en ce qui a trait aux éléments de la psychologie cognitive a fait ressortir des éléments forts intéressants. Premièrement, les étudiants interviewés ont indiqué que la formule de la classe inversée ne se prête pas à tous les cours. Ils ont soulevé le cours de mécanique des fluides dans lequel les développements théoriques sont particulièrement lourds (par exemple les équations de Bernoulli). Dans un tel contexte, ils doutent que la classe inversée puisse être efficace pour introduire un contenu théorique fort complexe :

«Par contre, quand il y a de gros fondements théoriques comme en mécanique des fluides, je pense que les lectures préalables seraient extrêmement compliquées. En fait, je n'aurais pas été capable d'apprendre Bernoulli par moimême avant d'arriver au cours. "

Ce témoignage illustre bien la notion de zone proximale de développement. Le contenu théorique que l'étudiant doit acquérir avant un cours doit donc être judicieusement dosé. Deuxièmement, les étudiants interviewés ont fait état de certains cours dont les séances les positionnent constamment en surcharge cognitive :

"Un autre cours qui pourrait bien bénéficier de la classe inversée est C'est de la grosse théorie pendant trois heures. Les PowerPoint font toujours environ 80 diapositives. Ensuite, il $y$ a les lectures à la maison, mais c'est trop, car je n'ai pas réussi à tout comprendre ce qui a été abordé dans les cours. »

En ce qui a trait à la taxonomie de Bloom, les étudiants interviewés ont apprécié pouvoir aborder en classe des problèmes cognitivement plus engageants, tout en ayant la possibilité de consulter le professeur en cas de besoin. Dans ces circonstances, le professeur doit pouvoir intervenir rapidement, mais dans un contexte où le nombre d'étudiants dans la classe est élevé, ceci peut s'avérer un défi :

"On devrait être plus souvent plongés dans des expériences similaires. J'aime le fait qu'on puisse aborder en classe des problèmes avancés. Vous étiez là pour nous aider et ça permet de confirmer que nous avons bien compris la matière. Par contre, c'était exigeant pour vous parce que vous vous promeniez partout pour répondre aux questions de toutes les équipes. »

Enfin, il y a eu les passages de l'entretien qui ne cadraient avec aucun des codes. Ils ont été regroupés dans un code intitulé "matériel résiduel". L'idée n'est pas d'exclure de l'analyse ce matériel, mais plutôt d'en faire une analyse interprétative purement inductive. En effectuant l'analyse du matériel résiduel, l'utilisation de capsules vidéo a été soulevée. Étonnamment, les étudiants interviewés n'ont pas manifesté un intérêt marqué envers cette façon de diffuser de l'information :

"C'est un couteau à double tranchant. Il y a des étudiants qui vont consulter les vidéos pour aller chercher un complément d'information. Par contre, les autres étudiants vont juste être encore plus lâches qu'ils ne le sont présentement en ne faisant aucune lecture. De mon côté, les capsules vidéo ne me parlent pas beaucoup. J'ai de la difficulté à bien être concentré et ça finit par être monotone. Il y a aussi moins d'interactions qu'une activité en classe. »

Cette information obtenue lors de l'entretien de groupe a une conséquence importante. Il était initialement prévu que des capsules vidéo allaient être produites pour la session d'automne 2019. À cause de ce manque d'engouement manifesté envers les capsules vidéo, il a été décidé de ne pas les produire dans l'immédiat et de consulter plus d'étudiants dans les prochains mois pour mieux comprendre la situation.

Dans un contexte de triangulation des données [4], la satisfaction des étudiants a été mesurée, à la fin de la session, à partir de l'évaluation institutionnelle du cours. Malgré qu'il s'agissait de la première fois où le cours MEC423 était offert en classe inversée, le professeur Terriault a obtenu sa meilleure évaluation pour ce cours depuis la première fois qu'il l'a enseigné en 2007, et cela avec une évaluation de 4,35/5 (moyenne départementale de 3,99/5 avec un écart type de 0,43).

Les données qualitatives et quantitatives collectées par le questionnaire, l'entretien de groupe et l'évaluation institutionnelle sont plutôt encourageantes et incitent la poursuite des efforts pour implanter la classe inversée. À 
court terme, plusieurs améliorations seront apportées aux cours dès l'automne 2019 dont les principales sont :

- Poursuivre l'utilisation des billets d'entrée, mais en réduisant leur pondération à $15 \%$ de la note finale,

- Abolir la correction des billets d'entrée par les pairs durant les séances de classe afin de dégager plus de temps pour des activités cognitivement engageantes,

- Ne pas aller de l'avant avec la production de capsules vidéo à l'été 2019 comme initialement prévu,

- Entamer les démarches afin que le cours soit offert dans une salle de classe dotée de bureaux et de tables facilement amovibles afin de pouvoir la configurer rapidement en mode de travail collaboratif.

\section{CONCLUSION}

Le projet de recherche décrit dans cette publication vise à étudier de manière rigoureuse l'efficacité de la classe inversée implantée dans un cours obligatoire de $1^{\text {er }}$ cycle en génie mécanique. Cette implantation est réalisée en étapes et dans cet article, les principaux constats suite à la première expérience de classe inversée sont rapportés. La voix des étudiants a été écoutée en collectant des données de trois façons différentes: un questionnaire rempli en classe, un entretien de groupe avec quelques volontaires et l'évaluation institutionnelle du cours.

Les donnes recueilles indiquent que l'expérience de la classe inversée a été positive pour les étudiants. Pour ce qui est du professeur, l'aventure s'est avérée nettement plus intéressante et enrichissante qu'un enseignement traditionnel magistral, et cela malgré les efforts colossaux qui ont été investis pour adapter le cours. Le projet de recherche visant à comparer de manière rigoureuse la classe inversée à une classe traditionnelle conserve donc son cap initial qui devrait culminer à l'automne 2020.

\section{Références}

[1] A. Abeysekera et P. Dawson, "Motivation and cognitive load in the flipped classroom: definition, rationale and a call for research," Higher education research and development, vol. 34, no. 1, pp. 1-14, 2015.

[2] C. Baribeau, "Analyse de données des entretiens de groupe," Recherches qualitatives, vol. 28, no., pp. 133148, 2009.

[3] M. Blais et S. Martineau, "L'analyse inductive générale : description d'une démarche visant à donner un sens à des données brutes," Recherches qualitatives, vol. 26, no. 2, pp. 1-18, 2006.
[4] M. Drapeau, "Les critères de scientificité en recherche qualitative," Pratiques psychologiques, vol. 10, pp. 79-86, 2004.

[5] A. Dumont et D. Berthiaume, La pédagogie inversée Enseigner autrement dans le supérieur avec la classe inversée. DeBoeck supérieur, 2016, 235 pp.

[6] C. Fortin, R. Rousseau, Psychologie cognitive: une approche de traitement de l'information. Presses de l'Université du Québec, 2015, 388 pp.

[7] H. Fry, S. Ketteridge, et S. Marshall, A Handbook for Teaching and Learning in Higher Education: Enhancing Academic Practice. Routledge, 2015 (4 ${ }^{\text {th }}$ ed.), 452 pp.

[8] D.R. Krathwohl, "A revision of Bloom's taxonomy: an overview," Theory into practice, vol. 41, no. 4, pp. 212$218,2002$.

[9] Z. Kzltepe, "Motivation and demotivation of university teachers," Teachers and Teaching: Theory and Practice, vol. 14, no. 5-6, pp. 515-530, 2008.

[10] J. Mukamurera, F. Lacourse et Y.Couturier, "Des avancées en analyse qualitative : pour une transparence et une systématisation des pratiques," Recherches qualitatives, vol. 26, no. 1, pp. 110-138, 2006.

[11] N. Nelson, "Flipping the engineering classroom," Actes de la 2014 Canadian Engineering Education Association conference (Canmore, AB, Canada, 8-11 juin 2014), Paper 090, 5 pp., 2014.

[12] R. Ryan et E. Deci, "Self-determination theory and the facilitation of intrinsic motivation, social development and well-being," American psychologist, vol. 55, no. 1, pp. 68$78,2000$.

[13] J. Sweller, "Cognitive load during problem solving: Effects on learning," Cognitive Science, vol. 12, pp. 257$285,1988$.

[14] R. Talbert, Flipped Learning: A Guide for Higher Education Faculty, Stylus Publishing, 2017, 264 pp.

[15] A. Tricot, L'innovation pédagogique. Éditions Retz, 2017, $160 \mathrm{pp}$.

[16] R. Viau, "La motivation : condition au plaisir d'apprendre et d'enseigner en contexte scolaire," Actes du $3^{e}$ congrès des chercheurs en éducation (Bruxelles, Belgique, 16-17 mars 2004), pp. 15-30, 2004.

[17] L.S. Vygotsky, Mind in society: The development of higher psychological processes. Harvard University Press, 1980, $176 \mathrm{pp}$ 